\title{
Prosthetic Leg
}

\author{
Aiswarya M R ${ }^{1}$, Athira K S ${ }^{2}$, Nijil Aziz ${ }^{3}$, Reshma Nathan ${ }^{4}$, Varsha T Joy ${ }^{5}$, Della Reasa Valiaveetil ${ }^{6}$ \\ UG Scholar, Electronics and Communication, IES College of Engineering, Thrissur, India ${ }^{1,2,3,4,5}$ \\ Asst. Professor, Electronics and Communication, IES College of Engineering, Thrissur, India ${ }^{6}$
}

\begin{abstract}
This research paper proposes the implementation of a system which can help the amputee torestore the utility of the lost limb by receiving electrical signal from the residual limb. Prosthetic devices substitute a missing or defective part of the body. Prosthetic devices have the ability to restore some mobility and functionality to the wearer. These can range from oral prostheses to limb prostheses. Limbs are often lost through trauma disease or a congenital disorder. Once the limb is removed, it can be very hard to resume to a normal life. Prosthetic limbs help give the amputee some sort of mobility that they would not have had without the device.A number of factors must be considered when selecting the right foot/feet for your lifestyle. These factors include amputation level, age, weight, foot size, activity level, goals and occupational needs. The focus of our research is to design a low-cost lower limb prosthetic, capable of knee movement in response to electrical signals from the residual limb.
\end{abstract}

Keywords: ATmega328, Arduino Promini 328, EMG sensor

\section{INTRODUCTION}

In today's world accidents are increasing day by day. The human body is a complex, but efficient machine. When it is at its best, it runs exactly like a well-lubricated machine. Each organ plays a very specific role, and when it is played to perfection, the machine runs smoothly. Almost all the organs and parts of the body (except the ones that have been rendered insignificant by evolution) are vital to its functioning. In some cases, owing to a birth defect, a disease, or an accident, a person might lose the function of one or more organs. The loss of function ranges from impaired ability to complete absence. In all such cases, one of the ways to make the life of the said personis referred to as an 'artificial organ'.In our system we uses aArduino/Genuino Uno which is a microcontroller board, based on the ATmega328Pand it is used in our design. The prosthetic reads the electrical signals from the residual limb and powers the motor for movement in accordance with the electrical signals. Here EMGsensor measures the electrical activity of muscles at rest and during contraction and bluetooth is used for wirelesscommunication. The readings are taken from the EMG sensor and send to prosthetic limb which are made to power the motors for the automation.

\section{RELATEDWORK}

\section{A. Brain Computer Interface}

Brain-computer interface (BCI) which uses electro physiological measures of brain function to enable individuals to communicate with the external world, by passing normal neuromuscular pathways. While it has been suggested that this control can be applied for neuroprostheses, few studies have demonstrated practical BCI control of a prosthetic device. In this paper, an electroencephalogram (EEG)-based motor imagery BCI is presented to control movement of a prosthetic hand. Hand was instrumented with force and angle sensors to provide haptic feedback and local machine control.

\section{B. Soft Orthosis}

There are human fingers and hands which are frequently injured because they are delicate, complex and used constantly.Morethan 3 million people in the United States suffer from hand or forearm disabilities and worldwide, hand injuries account for one-third of all work injuries. Due to the importance of hands and the prevalenceof hand issues, there is an increasing effort toward developing hand orthotics. These efforts have resulted in active hand orthoses that have been used for rehabilitation training and restoring partial hand function. To ensure safety and toreduce control complexity, some orthoses use mechanical compliances, such as under actuated linkages or low-stiffness materials and structures (e.g., rubbers and flexible wires). The orthoses made of elastomeric materials tend to be more comfortable, perhaps because their low elastic modulus is similar to that of human skin.

\section{C Leg}

The best known present day artificial leg is the c-leg produced by otto bock. The user can choose between two modes: one for walking and one for bicycling (or any other programmed activity). The problem is switching between modes, as 
the wearer as to swing the leg forward in a jerky fashion. The c-leg supports 10 programmable mode, switchable through a small remote control device about the size of a car alarm remote.An inbuilt microprocessor interprets the user's movement and anticipates their actions, allowing motion changes in real time. The system is actuated through the leg's hydraulic movement. It gives users greater flexibility to change speed or direction without scarifying stability. The 10 switchable modes enable various sports activities, including cycling, running, natural driving and other programmable activitiesthat require different leg actions to normal walking and star climbing. Current challenges are to stretch the unit's battery life to 50 hours on one charge and to make the leg salt-water resistant. These are both requirements to support military use.The advantage of c-leg technology have been investigated in detail in numerous scientific studies. Among other things, these studies have proven that the frequency of falling is much lower with the cleg compared to other leg prosthesis system. A c-leg user also does not have to concentrate on the prosthesis all the time. This permits greater everyday freedom of movement.

\section{PROPOSEDMETHODOLOGY}

Body powered prosthetics are operated by multiple cables and are connected to the residual portion of the body. The advantage of these is that they do not require any electrical power supply. The main disadvantage to these type of prosthetics is that they require an unnatural movement in order to operate them to perform a task. So we introduced a myoelectric prosthetic lower limb.Surface electrodes are placed on the skin of the residual limb to pick up the signals that are transmitted down the nerves to this limb. This type of prosthetic can often allow the user multiple degrees of freedom, DOF, meaning multiple movements can often be made simultaneously and within a smooth succession.

\section{BLOCKDIAGRAM}

The block diagram of the proposed system is given in Figure.1. The power supply provides a regulated DC voltage. Myoelectricsignals are produced by a muscle whenit contracts. Wave receptors receive impulse from the muscle and transfer it to EMG module. ATmega368p microcontroller readthe input and control the output. Arduino is a platform based program coding software. Through bluetooth, the datas are transmitted.

At the receiver section (Figure 1(b)), the data is received through another Bluetooth module and converted in to corresponding signals for the servomotor to work according to the input.

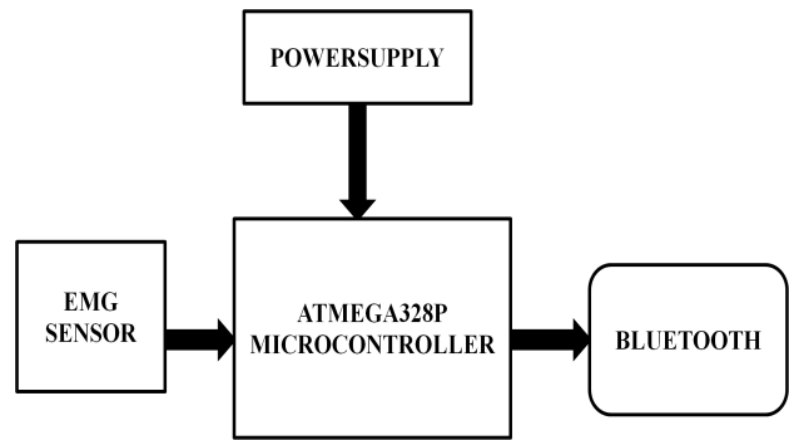

Fig.1(a)Transmitter system block diagram

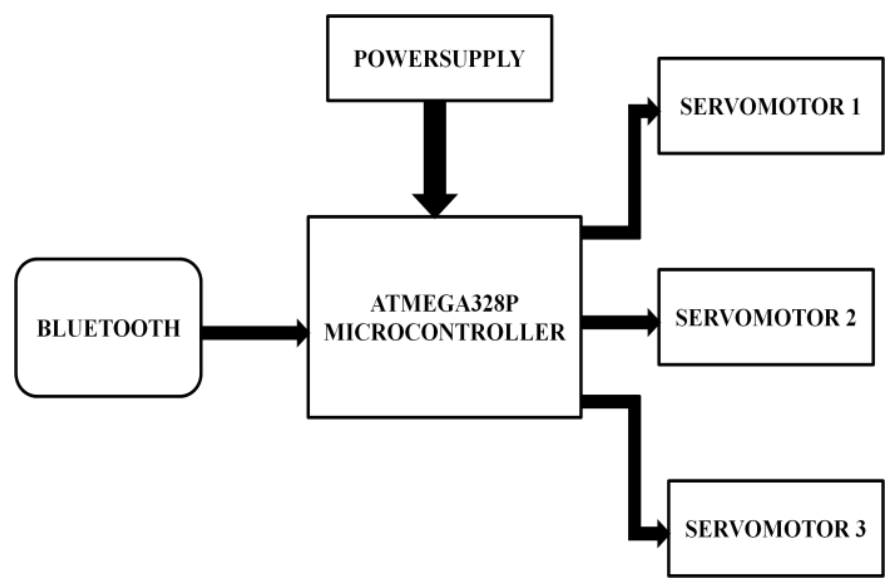

Fig.1(b)Receiver system block diagram 
Vol. 5, Issue 4, April 2017

\section{HARDWARE IMPLEMENTATION}

EMG is a completely non-invasive technology that allows you to easily place EMG electrodes with stickers to the skin. No muscle innervation is necessary, which renders EMG an ideal method for monitoring physiological processes without interfering other established routines and movement patterns. The Arduino Pro is a microcontroller board based on the ATmega328.It has inbuilt ADC and USART data transfer module, that helps in the analysing the analog signals obtained from the emg sensors. The microcontroller is programmed using ARDUINO Uno board. Figure 2(a) shows the emg sensors placed on the muscle and Figure 2(b) shows the overall experimental setup of our system.

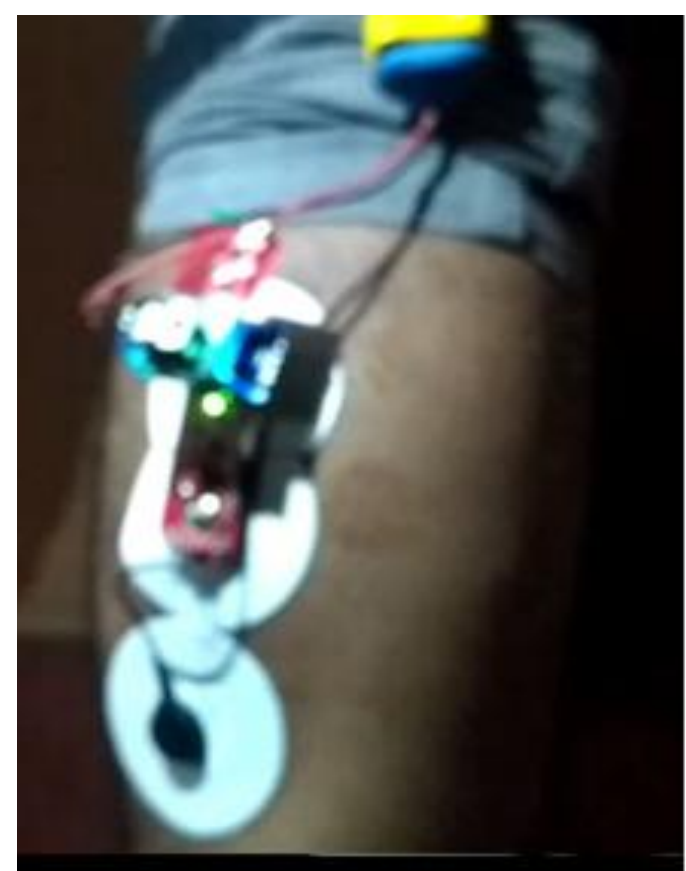

Fig.2(a) EMG sensors placed on muscles

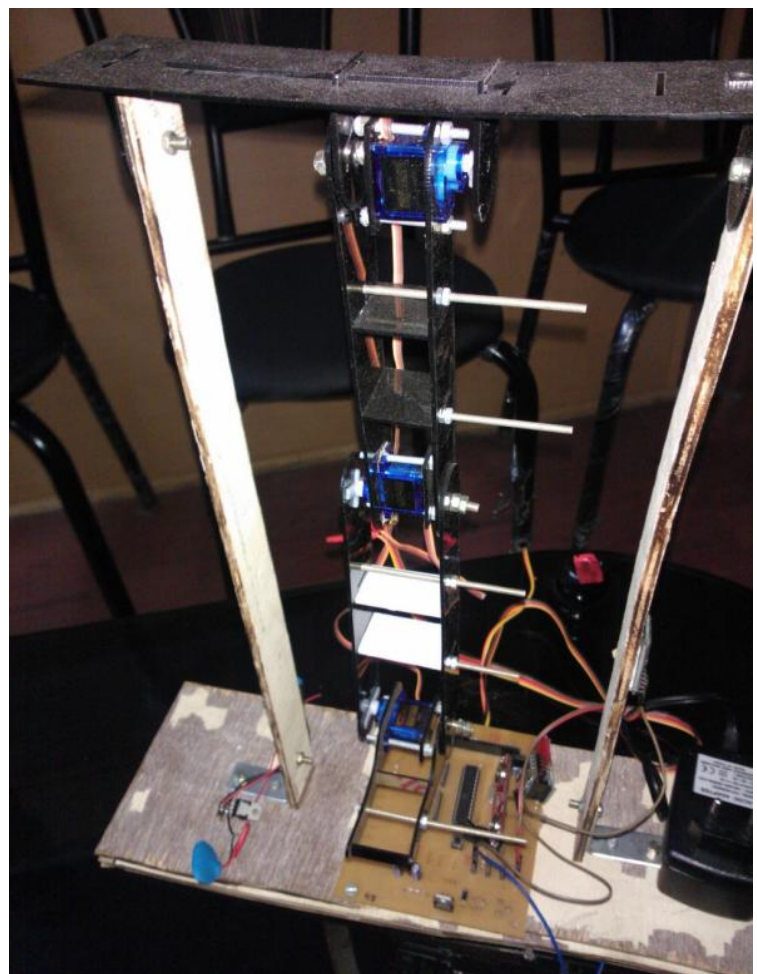

Fig.2(b) Overall experimental setup 


\section{International Journal of Innovative Research in} Electrical, Electronics, Instrumentation and Control Engineering

ISO 3297:2007 Certified

Vol. 5, Issue 4, April 2017

\section{SOFTWARE IMPLEMENTATION}

The system software was developed using the following software tools. ARDUINO IDE Version 1.7.6 - to program the microcontroller.

A. Flow chart for System hardware

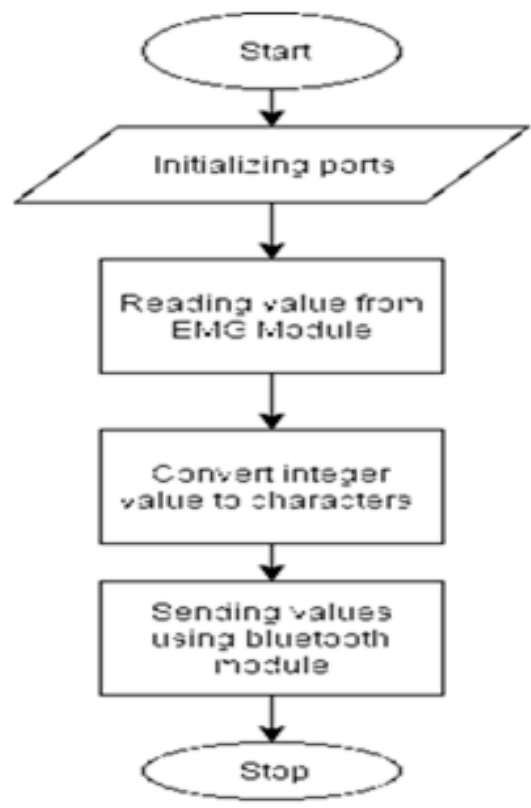

Fig.3(a) Transmitter section

In transmitter section, the ATmega microcontroller reads the values from the emgsensors.EMG module consists of signl amplifier and optimizer. Optimizer compare the three signals and gives out a corresponding analog value which ranges from 0-1023. These values are then converted into characters and send using Bluetooth module. This process is depicted in Fig. 3(a).

In the receiver section, using another bluetooth module data are received and the ATmega microcontroller reads the character values and controls the servomotors fixed at the hip, knee and ankle. This process is depicted in Fig.3(b).

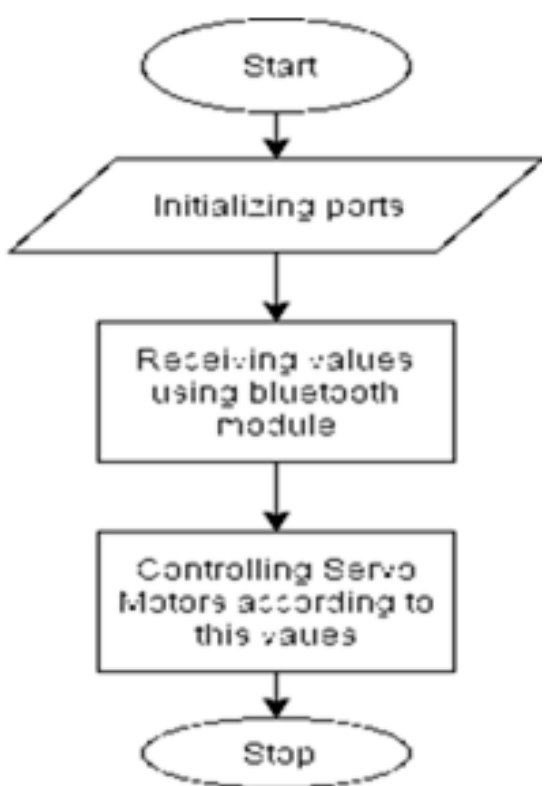

Fig.3(b) Receiver Section 
B. System Software Simulation Model

The data sent from the EMG module is stored and analysed for the approximate values setting on the programming section.

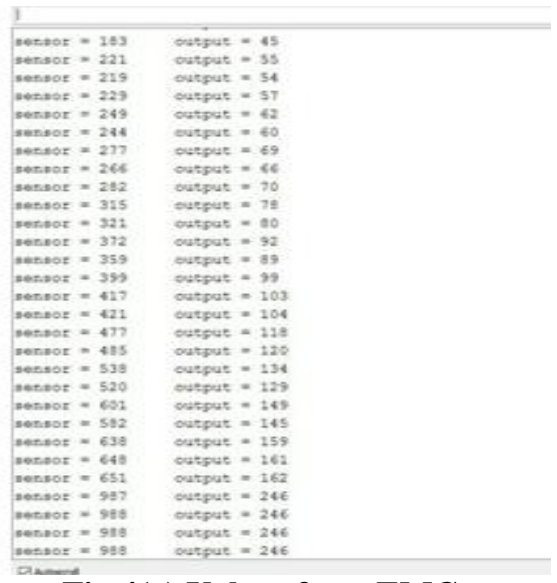

Fig.4(a) Values from EMG

While walking maximum hip movement angle is 30 degree. The analog value which is from $0-1023$ scales 0 to 30 degree. So when the muscle is in resting state the analog value will be 0 and the corresponding angle will be 0 degree. And when the muscle generate maximum impulse, the analog value will be 1023 and the corresponding angle will be 30 degree. Maximum knee movement angle is 10 degree, the analog value which is from 0-1023 scales 0-10 degree. So when the muscle is in resting state the analog value will be 0 and the corresponding angle will be 0 degree. When muscle generate maximum impulse the analog value will be 1023 and the corresponding angle will be 10 degree. Maximum ankle movement is 30 degree. The analog value which is from 0-1023 scales 0-30 degree. So when the muscle is in resting state the analog value will be 0 and the corresponding angle will be 0 degree. When muscle generate maximum impulse the analog value will be 1023 and the corresponding angle will be 30 degree.

\section{CONCLUSION}

Prosthetic Leg is one of the latest advancements in an evolving technological world. They allow the control of prosthetic limb through the activity of electrical signals from the muscles of the leg. Unlike older prosthesis, this technology allows for much more control of the lower limb.Looking towards the future myoelectric prosthesis is evolving even further to incorporate a new technology that will allow signals to come straight from nerves themselves.

\section{ACKNOWLEDGEMENT}

The authors would like to acknowledge the Management Principal, Head of the department, all the faculties and staff of Electronics and Communication Department, of IES College of Engineering, Thrissur, Kerala, for their aid and technical guidance given during the entire course to complete it providentially. Also we extend our sincere thanks to the entire R \& D team of the college for giving the facility to do our project in the R \& D lab.

\section{REFERENCES}

[1] EnglehartK."Electromyogram pattern recognition for control of powered upper-limb prostheses: State of the art and challenges for clinical use".Journal of Rehabilitation Research and Development 2011

[2] Barto, Taylor."Designing Electronics for an Artificial Leg".The Downtown Review.Vol. 1.Iss.1 (2015).

[3] F. Sup, H. Varol, J. Mitchell, T. Withrow, and M. Goldfarb, "Design and Control of an Active Electrical Knee and Ankle Prosthesis," IEEE International Conference on Biomedical Robotics and Biomechatronics, Scottsdale, AZ, pp. 523-528, 2008.

[4] Rainoldi, G. Galardi, L. Maderna, G. Comi, L. Lo Conte, R. Merletti, "Repeatability of surface EMG variables during voluntary isometric contractions of the biceps brachiimuscle",Journal of Electromyography and Kinesiology 9 (1999) 105-119

[5] Didier Staudenmann, IdsartKingma, Andreas Daffertshofer, Dick F.Stegemanand Jaap H. van Dieën,"Improving EMG-Based Muscle ForceEstimationbyUsing a High-Density EMG Grid and Principal Components Analysis”, IEEE Transactions On Biomedical Engineering, vol. 53, no. 4,April 2006

[6] De Stefano, J.H.Burridge, V.T. Yule, R. Allen, "Effect of gait cycle selection onEMG analysis during walking in adults and children with gait pathology" Gait andPosture (2004) 92-101

[7] "Myoelectric Prosthetics 101" Ottobock USA. OttobockGroup, n.d.Web. 6 Oct. 2015 


\section{International Journal of Innovative Research in} Electrical, Electronics, Instrumentation and Control Engineering

ISO 3297:2007 Certified

Vol. 5, Issue 4, April 2017

\section{BIOGRAPHIES}

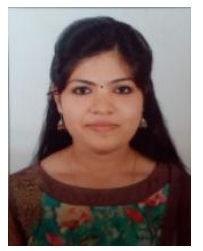

Aiswarya M R currently pursuing the Bachelores degree in Electronics and Communication Engineering from the IES College of Engineering, Thrissur. She was an active member of NSS IES for years 2014-16.

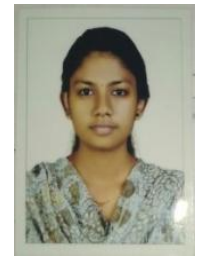

Athira K S currently pursuing the Bachelor ${ }^{\mathrm{ee}}$ s degree in Electronics and Communication Engineering from the IES College of Engineering, Thrissur.

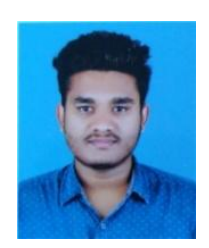

Nijil Aziz currently pursuing the Bachelores degree in Electronics and Communication Engineering from the IES College of Engineering, Thrissur.

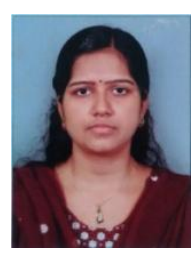

Reshma Nathan currently pursuing the Bachelor"s degree in Electronics and Communication Engineering from the IES College of Engineering, Thrissur.

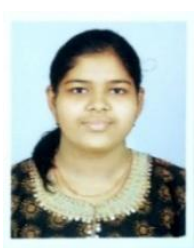

Varsha T Joy currently pursuing the Bachelore's degree in Electronics and Communication Engineering from the IES College of Engineering, Thrissur.

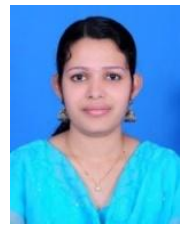

Della ReasaValiaveetil Working as Assistant Professor IES College of Engineering, completed MTECH from Hindustan College of Engineering \& Technology and BTECH from Sahrdaya College of Engineering \& Technology. 